\title{
应激对大鼠腹腔巨唻细胞释放 过氧化氢的抑制作用*
}

范少光丁玄宙 丁桂风** 靽济生

(北京医科大学生理教研室、**免度学教研公)

人们已经发现应激可以抑制 $\mathrm{T}$ 淋巴细胞 ${ }^{[1,2]}$ 和天然杀伤细胞 ${ }^{[3]}$ 的功能. 应激对 $\mathrm{B}$ 淋巴细胞 的作用比较复杂，根据应激的性质和强度不同，以及动物的生物学特性不同，或产生抑制作用， 或产生加强作用 ${ }^{[4,5]}$. 巨噬细胞 ( $\left.M \phi\right)$ 在免疫系统接受刺激、传递信息过程中起着重要作用. 其本身释放过氧化氢 $\left(\mathrm{H}_{2} \mathrm{O}_{2}\right)$ 、超氧离子 $\left(\cdot \mathrm{O}_{2}^{-}\right)$及羟基游离基 ( $\left.\mathrm{HO} \cdot\right)$ 对微生物及肿瘤 细 胞具有杀伤作用 ${ }^{(6)}$. 然而, 应激对 $\mathrm{M} \phi$ 释放 $\mathrm{H}_{2} \mathrm{O}_{2}$ 的作用至今未见报道. 本文观察了应激对 大䦠腹腔 $\mathrm{M} \phi$ 释放 $\mathrm{H}_{2} \mathrm{O}_{2}$ 功能的影响, 并对其作用机理进行了初步探讨.

\section{一、材料与方法}

1. 应做摸瑟取 $170-210 \mathrm{~g} \mathrm{SD}$ 纯系大鼠，雌雄兼配. 置动物于特制塑料筒内, 两后肢: 伸出筒外，插入两对不锈钢电极，使用 WQ-6D 电刺激仪给与电刺激。电刺激的波宽为 $0.3 \mathrm{~ms}$ 双向疏密脉冲, 频率为 2-15 Hz. 电刺激仪恒压输出 4 或 $6 \mathrm{~V}^{[7]}$. 实验组大鼠给与 $4 \mathrm{~V}$ 电刺激: $10 \mathrm{~h}$ 及 $6 \mathrm{~V}$ 电刺敫 $10 \mathrm{~h}$ (共 $20 \mathrm{~h}$ ). 对照鼠仅置于筒中不给与刺激.

2. 纳曲围 (Naltrexone, NTX) 注射 NTX 溶于生理盐水, 皮下注射两次, 每次 10 $\mathrm{mg} / \mathrm{kg}$. 应激开始时注射一次, 应激 $10 \mathrm{~h}$ 注射另一次.

3. 淋巴细胞转化实检 20 只大鼠均分两组, 应激实验后以乙醚轻度麻醉动物, 心肚取 血 $2 \mathrm{ml}$. 以聚蔗糖(Ficoll)-泛影萷胺（Urografin）分层液(比重 1.077) 密度梯度离心法分离 出淋巴细胞, 以 Hanks' 液洗细胞两次. 分别以 RPMI 1640 细胞培养液(含 $10 \%$ 小牛血清)将 细咆制成 $2 \times 10^{6}$ 细胞 $/ \mathrm{ml}$ 的细胞悬液,然后注入 96 孔细胞培养板内,每孔 $200 \mu \mathrm{l}$. 使用不同 浓度的刀豆素 $\mathrm{A}(\mathrm{ConA}, 0.625,1.25,2.5,5,10 \mu \mathrm{g} / \mathrm{ml})$ 刺激淋巴细胞转化. 将细胞放人 $\mathrm{CO}_{2}$ 解育箱内 (含 $95 \%$ 空气 $5 \% \mathrm{CO}_{2}$ ) $37{ }^{\circ} \mathrm{C}$ 捊育 $72 \mathrm{~h}$. 餒育完毕每孔加入 ${ }^{3} \mathrm{H}$-胸腺嘧啶核苷 ( $3 \mathrm{H}-\mathrm{TdR}$, 比活 $20 \mathrm{Ci} / \mathrm{mmol}$ ) $0.2 \mu \mathrm{Ci} / 20 \mu \mathrm{l}$, 继续榎育 $6 \mathrm{~h}$ 后收获细胞, 以液闪计数器测定 ${ }^{3} \mathrm{H}$ 掺人最.

4. $\mathrm{M}_{\phi}$ 释放 $\mathrm{H}_{2} \mathrm{O}_{2}$ 测定 分别在应激前后以乙醚轻度麻醉大鼠，以 $\mathrm{Hanks}^{\prime}$ 液灌洗腹 腔收集 M $\phi$. 分别计数并分类细胞. 以 RPMI 1640 细胞培养液将细胞制成 $2 \times 10^{6}$ 细胞 $/ \mathrm{ml}$

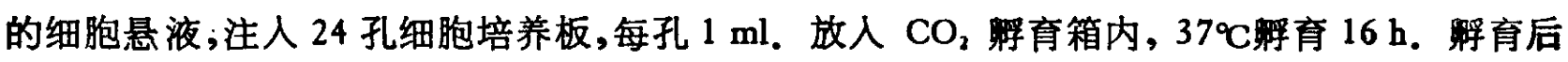
弃去上清液, 并以淢萄糖磷酸缓冲液 ( KRPG, 含 $5.5 \mathrm{mmol} / 1$ 葡萄糖) 洗去未贴壁细胞.

在体外实验中, 从正常六鼠腹腔灌洗液中收集 M . 以 RPMI 1640 液制成 $2 \times 10^{6}$ 细

本文1987年11月26日收到。

- P.生部、教育委员会、国家自然科学基金觉助项目. 
胞 $/ \mathrm{mi}$ 的组咆悬液,注人 24 孔细胞培养板, 每孔 $1 \mathrm{ml}$, 并分别与 $10^{-12}-10^{-6} \mathrm{~mol} / 1$ 浓度范围 的 $\beta$-仢啡状 ( $\beta$-endorphin, $\beta$-End，Sigma 公司）、吗啡(中国药剂公司)、亮-脑啡肽 (Leuenkephalin, Leu-Enk, Sigma 公司) 和强啡肽 A(1-13) (Dynorphin A, Dyn A, 由 A. Goldstein 教授赠送)其闰卵育 $20 \mathrm{~h}$.

以苂光分光光度法间接测定贴壁 $\mathrm{M} \phi$ 释放 $\mathrm{H}_{2} \mathrm{O}_{2}$ 量 ${ }^{[8]}$. 测定过程简述如下,腹腔细胞经胋 壁培养后,齐去上清液,以 Hanks' 液洗细胞两次. 然后每孔加 KRPG $1.5 \mathrm{ml}$, 内含十四酰佛波

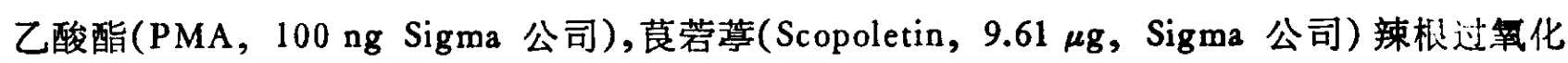
物酶（HRP，10 u, Sigma 公司). 卯育 $1 \mathrm{~h}$ 后，在发射波长 $370 \mathrm{~nm}$, 激发波长 $462 \mathrm{nmi}$ 下测 定各孔苂光强度, 并计算出各组 $\mathrm{M} \phi$ 释放 $\mathrm{H}_{2} \mathrm{O}_{2}$ 量.

5. $\beta$-End 测定应激反应后, 心脏取血,加肝素抗凝， $1000 \times g$ 离心分离血浆. 以放 射免疫测定方法 ${ }^{[9]}$ 分别测定实验鼠及对照鼠血浆 $\beta$-End 含量.

6. 统计学分析方法 连续性资料分析采用方差分析法 (ANOVA) 统计两组间显著性 差异. 两两比较采用 $\mathrm{t}$ 检验统计方法.

\section{二、结 果}

10 只对炤组大鼠外周血淋巴细胞与不同剂量 ConA 共同捊育后, 其 ${ }^{3} \mathrm{H}-\mathrm{TdR}$ 摄人值随: ConA 浓度增大而升高，有良好的剂量效应关系. 10 只经过应激刺激后的大鼠，其淋巴细胞 摄取 3H-TdR 能力明显受到抑制. 在 ConA 浓度为 5 及 $10 \mu \mathrm{g} / \mathrm{ml}$ 时, 对炤组和实验组 'H$\mathrm{TdR}$ 的掺入量分别为 $15551 \pm 5008,21641 \pm 6621$ 和 $2289 \pm 765,5003 \pm 1219 \mathrm{cpm}$.

10 只对照大鼠放入塑料筒内前后腹腔 $\mathrm{M} \phi$ 释放 $\mathrm{H}_{3} \mathrm{O}_{2}$ 最分别为 $23.3 \pm 1.59 \mathrm{nmol} / 2 \times$ $10^{6}$ 细胞 $\cdot h$ 和 $21.8 \pm 1.70 \mathrm{nmol} / 2 \times 10^{6}$ 细胞 $\cdot h$, 无明显变化. 然而 10 只实验大鼠在应激 反应后其 $\mathrm{M} \phi$ 释放 $\mathrm{H}_{2} \mathrm{O}_{2}$ 量为 $9.7 \pm 0.9 \mathrm{nmol} / 2 \times 10^{6}$ 细胞 $\cdot h$, 明显低于应激前的释放量 $\left(21.0 \pm 1.30 \mathrm{nmol} / 2 \times 10^{6}\right.$ 细胞 $\left.\cdot h, p<0.001\right)$. 与对照鼠柏比, $\mathrm{M} \phi$ 释放 $\mathrm{H}_{2} \mathrm{O}_{2}$ 能力明显 受到抑制(图 1)。应激抑制大鼠腹腔 $\mathrm{M} \phi$ 释放 $\mathrm{H}_{2} \mathrm{O}_{2}$ 呈明显时效关系(图 2).

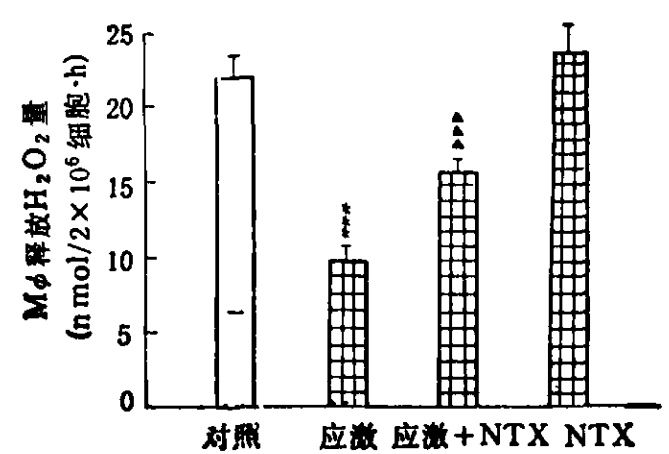

图 1 应激对 $\mathrm{M} \phi$ 释放 $\mathrm{H}_{2} \mathrm{O}_{2}$ 的影响及纳曲再 (NTX) 的对抗效应

试验动物数 $\mathrm{n}=10$, 应淌 $+\mathrm{N} 7 \mathrm{X}: 10 \mathrm{mg} / \mathrm{kg} \times 2$, NTX: $10 \mathrm{mg} / \mathrm{kg}_{\mathrm{g}} \times 2$.***表示与对盟组比较, $f<0.001 ; \Delta \Delta \Delta$ 表示与应滶组比较， $p<0.001$

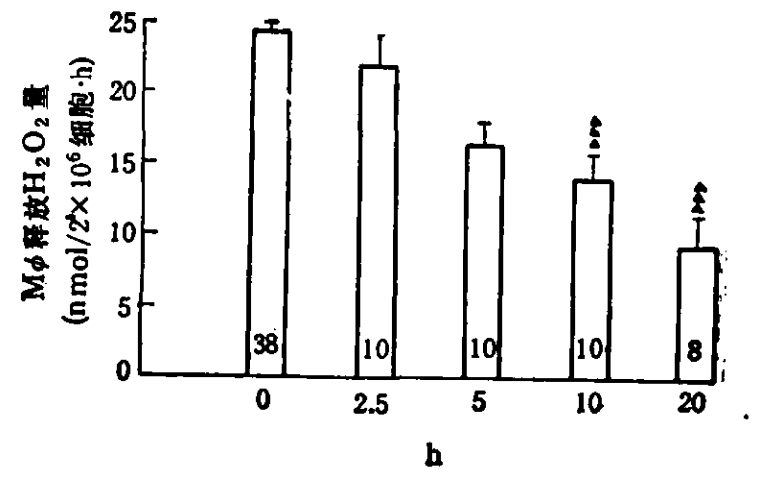

图 2 应激抑制大鼠腹腔 $\mathrm{M} \phi$ 释放 $\mathrm{H}_{2} \mathrm{O}_{2}$ 的时效关系

柱内数字表示动物数. $\Delta \Delta \Delta$ 表示与对耍 组相比, $p<0.001$

单纯注射 NTX 对大鼠腹腔 $\mathrm{M} \phi$ 释放 $\mathrm{H}_{2} \mathrm{O}_{2}$ 无明显影响. 对照组为 $20.8 \pm 1.68 \mathrm{nmol} /$ $2 \times 10^{\prime}$ 细胞 $\cdot h, N T X$ 组为 $23.6 \pm 2.04 \mathrm{nmol} / 2 \times 10^{6}$ 细胞 $\cdot h$. 而 $N T X$ 可部分转应败 
对 $\mathrm{M} \phi$ 释放 $\mathrm{H}_{2} \mathrm{O}$, 的抑制效应为 $15.5 \pm 0.9 \mathrm{nmol} / 2 \times 10^{3}$ 细胞 $\cdot \mathrm{h}(p<0.001$, 图 1).

实验组大鼠应激反应后其血浆 $\beta$-End 的含量 $(255.2 \pm 66.71 \mathrm{Pg} / \mathrm{ml})$ 明显高于对照组大 鼠的血㭚含量 $(85.6 \pm 28.44 \mathrm{Pg} / \mathrm{ml})$.

使用四种不同的阿片类药物与正常大鼠腹腔 $\mathrm{M} \phi$ 体外共同率育 $20 \mathrm{~h}$, 发现 $\beta$-End 在浓度 为 $10^{-12} 、 10^{-10} 、 10^{-8}$ 及 $10^{-6} \mathrm{~mol} / 1$ 时表现出明显地抑制 $\mathrm{M} \phi$ 释放 $\mathrm{H}_{2} \mathrm{O}_{2}$ 效应. 实验组与对炤组 $\mathrm{H}_{2} \mathrm{O}_{2}$ 释放量分别为 $22.6 \pm 0.3,21.3 \pm 0.2,19.9 \pm 0.3,19.2 \pm 0.3$ 和 $24.8 \pm 0.2,24.6 \pm 0.2,24.5 \pm$ $0.3,24.8 \pm 0.2 \mathrm{nmol} / 2 \times 10^{6}$ 细胞 $\cdot h$. 吗啡只在高浓度情况下 $\left(10^{-6} \mathrm{~mol} / 1\right)$ 表现出抑制作用, 而 Dyn 和 Leu-Enk 在 $10^{-12}-10^{-6} \mathrm{~mol} / \mathrm{J}$ 浓度范围内对 $\mathrm{M} \phi$ 释放 $\mathrm{H}_{2} \mathrm{O}_{2}$ 无明显影响 (图 3). 将 $\operatorname{NTX}\left(10^{-6} \mathrm{~mol} / 1\right)$ 和 $\beta$-End $10^{-12}-10^{-6} \mathrm{~mol} / 1$ 共同与 $\mathrm{M} \phi$ 体外摡育 $20 \mathrm{~h}$, $\beta$-End 对 $\mathrm{M} \omega$ 释放 $\mathrm{H} . \mathrm{O}$ ，的抑制效应被完全翻转 (图 4).

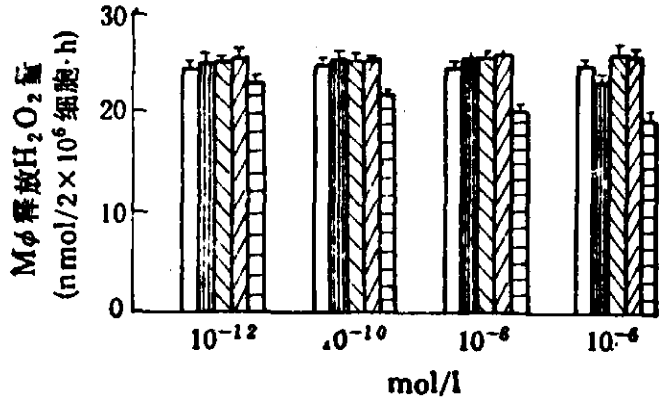

图 3 阿片样药物在体外对 $\mathrm{M} \phi$ 释放 $\mathrm{H}_{2} \mathrm{O}$, 的影响

ANOVA 统计结果表明 $\beta$-End 组与对照组具 有明显. 美异 $(p<0.01)$.

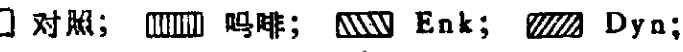
戸 $\beta$-End; $n=5$

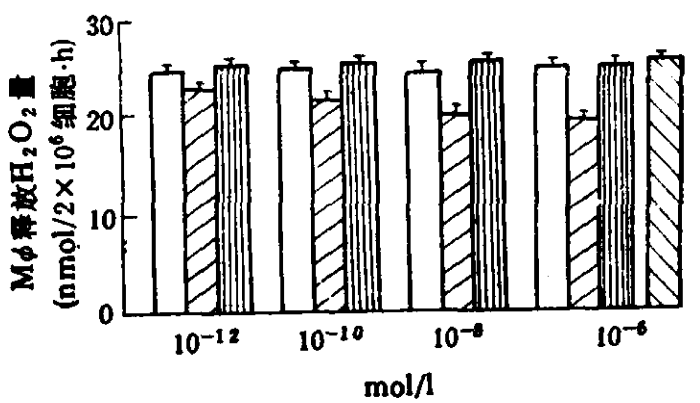

图 4 纳曲，洞 (NTX) 对 $\beta$-End 抑制 $M \phi$ 释放 $\mathrm{H}_{2} \mathrm{O}_{2}$ 的对抗作用

ANOVA 统计结果表明 NTX 组与单纯 $\beta$-End 组 具有明显差异 $(p<0.01)$.

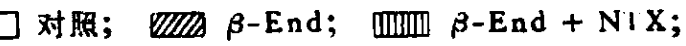
$\triangle \mathrm{NTV}, n=5 \cdots 10$

\section{三、讨 论}

以往的实验结果已经证实大鼠在电刺激造成的应激反应后其 $\mathrm{T}$ 淋巴细胞转化功能受到抑 制 $^{[1,2]}$, 我们的实验亦得到了相同的结果. 本实验进一步观察 $\mathrm{T}$ 淋巴细胞功能在应激反应中受 到抑制的同时, 其它免疫细胞的功能是否也发生变化, 我们首先观察了 $\mathrm{M} \phi$ 功能的变化,结果 表明,大鼠在应激反应时 $\mathrm{M} \phi$ 释放 $\mathrm{H}_{2} \mathrm{O}_{2}$ 功能也明显受到抑制.

目前已有不少关于应激对免度系统产生抑制效应机理的报告. Riley ${ }^{[10]}$ 证实应激对免疫系 统产生影响的主要介导物质是肾上腺分必的激素. Keller 等 ${ }^{[2]}$ 提出应激对 $\mathrm{T}$ 淋巴细胞转化功 能的抑制作用并非完全由肾上腺系统介导完成. Shavit 等 ${ }^{[3]}$ 认为阿片样物质可能参与电刺 激 抑制大鼠自然杀伤细胞产生细胞毒的功能. 我们在体外实验中发现, 生理浓度范围内的 $\beta$-End 即可对 $M \phi$ 产生抑制效应. 为了进一步证实这一结果, 我们使用 Pick 和 Keisari ${ }^{[11]}$ 建立的另一种 $\mathrm{H}_{6} \mathrm{O}_{2}$ 测定方法同样证实了 $\beta$-End 在体外对 $\mathrm{M} \phi$ 释放 $\mathrm{H}_{2} \mathrm{O}_{2}$ 的抑制效应. 已 有报道发现应激反应可以导致 $\beta$-End 血浆浓度升高 ${ }^{[22]}$. 我们在给与大鼠应激反应以后测定 了血浆 $\beta$-End 含量, 得到了相同的结果. 这些结果提示 $\beta$-End 在应激反应时 $\mathrm{M} \phi$ 释放 $\mathrm{H}_{2} \mathrm{O}$ ，的抑制效应中可能起有重要作用。

将 NTX 注射大鼠体内仅能部分对抗应激对 $\mathrm{M}_{\phi}$ 释放 $\mathrm{H}_{2} \mathrm{O}_{2}$ 的抑制效应, 这一点提示应 激对 $\mathrm{M} \phi$ 的抑制效应不仅由 $\beta$-End 所介导,可能是多因素介导的反应. 
$\beta$-End 是一种含有 31 个氨基酸的肽类物质,它可以与多种不同的阿片及非阿片类受体相 结合. 介导免疫功能发生变化的 $\beta$-End 受体可能不止一种类型,包括阿片样受体 ${ }^{[23]}$ 和非阿版

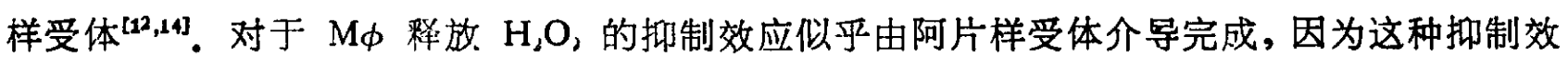
应不论在体们还是在体外都可被 NTX 所对抗. 我们在另一实验中, 应用应激后大鼠的腹腔 灌洗液培养正常大鼠 $\mathrm{M} \phi$, 与未应激鼠的腹腔灌流液相比, 具有显著抑制 $\mathrm{M} \phi$ 释放 $\mathrm{H}_{2} \mathrm{O}_{2}$ 的 能力, 而且旺明显剂效关系(待发表)。这一结果不仅再次证实应激对 $\mathrm{M} \phi$ 释放 $\mathrm{H}_{2} \mathrm{O}_{2}$ 的抑制 作用,而且提示在应激中腹腔液的成分有某些改变.

Sharp 等 ${ }^{[15]}$ 报告 $\beta$-End 和强啡肽 (Dyn) 在卯育的早期 (3 及 $15 \mathrm{~min}$ ) 可以短暂刺激人 腹腔 $\mathrm{M} \phi$ 和多形核白细胞释放超羍阴离子 $\left(\cdot \mathrm{O}_{2}^{-}\right)$. $\cdot^{-} \mathrm{O}_{2}^{-}$在超氧化物歧化酶的作用下可以转 变为 $\mathrm{H}_{2} \mathrm{O}_{2}^{[11]}$, 通常二者有平行变化的关系. 我们所观察到的 $\mathrm{M} \phi$ 释放 $\mathrm{H}_{2} \mathrm{O}_{2}$ 的抑制现象看 来不是由于应激或 $\beta$-End 首先刺激 $\mathrm{M} \phi$ 释放 $\mathrm{H}_{2} \mathrm{O}_{2}$, 经长时间应激或与 $\beta$-End 培养后使 $\mathrm{H}_{2} \mathrm{O}_{2}$ 耗竭所至. 因为从体内实验看, $\mathrm{H}_{2} \mathrm{O}_{2}$ 的释放抑制呈明显时效关系, 末发现先升高后降低 的现象. 在体外实验中, 我们将 $\beta-E n d ~\left(10^{-6} \mathrm{~mol} / 1\right)$ 以及应激后的腹腔灌洗液与正常 $\mathrm{M} \psi$ 培养 $20 \mathrm{~h}$, 同时加入测定系统反应液, 但不加细胞膜活化剂 PMA, 其释放的 $\mathrm{H}_{2} \mathrm{O}_{2}$ 量极微 $(0.55 \pm$ 0.015 和 $0.71 \pm 0.015 \mathrm{nmol} / 2 \times 10^{\mathrm{s}}$ 细胞 $\cdot \mathrm{h}$ ), 与相应对炤组 (无 $\beta$-End, $0.61 \pm 0.03 \mathrm{nmol} /$ $2 \times 10^{6}$ 细胞 $\cdot h$; 或正常大鼠腹腔灌洗液 $0.69 \pm 0.01 \mathrm{nmol} / 2 \times 10^{\circ}$ 细胞 $\cdot h$ ) 无明显差别. 看 来在我们的实验条件下应激和 $\beta$-End 确能抑制大鼠 $\mathrm{M}_{4}^{\prime}$ 释放 $\mathrm{H}_{2} \mathrm{O}_{2}$ 的能力. Sharp 等与 我们的结果分歧原因尚不清楚，可能与测定方法、时间、测定对象不同有一定关系。

Dyn 和 [D-Ala $\left.{ }^{2}, \mathrm{D}-\mathrm{len}^{5}\right]$ Enk 在体外对 $\mathrm{M} \phi$ 释放 $\mathrm{H}_{2} \mathrm{O}_{2}$ 无明显影响, 说明它们对 $\mathrm{M} \phi$ 可能没有直接作用. 这一阴性结果尚不能排除 Dyn 和 Enk, 在体外信育过程中被酶的 可能性,所以仍需要进一步的实验证实这两种肽对 $\mathrm{M} \phi$ 的影响.

综上所述, 本文实验结果证明: (1) 大鼠经电刺激造成的应激反应后, 腹腔 $\mathrm{M} \phi$ 释放 $\mathrm{H}, \mathrm{O}_{2}$ 能力下降; (2) 这种抑制效应的可能机制之一是应激诱导了血浆 $\beta$-End 含量升高, 从 而抑制了 $\mathrm{M} \phi$ 释放 $\mathrm{H}_{2} \mathrm{O}_{2}$ 的能力; (3) $\beta$-End 对 $\mathrm{M} \phi$ 的抑制效应可能是通过阿片受体介导. 完成.

致谢：感谢 L. L. Iversen 博士和 A. Goldstein 教授为我们提供 Dyn 和 $\beta$-End 药品.

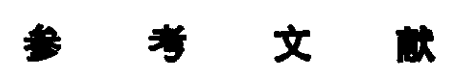

[1] Weiss, J. M. et al., Science, 213(1981), 1397-1400.

[2] Keller, S. E. et al., Science, 221(1983), 1301-1304.

[3] Shavit, Y. et al., Science, 223(1984), 188-190.

[4] Monjan, A. A. and Collector, M. I., Science, 196(1977), 307-308.

[5] Jaosoo, A. and Mekeuzic, J. M., Int. Arch. Allergs Appl. Immunol, 50(1976), 659-663.

[6] Klebanoff, S. J., Ann. Int. Med., 93(1980), 480-485.

[7] Han, J. S. et al., Pain, 27(1986), 101-115.

[ 8 ] Nathan, C. F. and Raot, R. K., J. Exp. Med, 146(1977), 1648-1662.

[9] Rossier, J. et al., Life Sci., 21(1977), 847-852.

[10] Riley, V., Science, 212 (1981), 1100-1102.

[11] Pick, E. and Keisari, Y., J. Immunol. Meth, 38(1980), 161-170.

[12] Gilman, S. C. et al., Proc. Nattl. Acad. Sci. USA, 79(1982), 4226-4230.

[13] De Carolis, C. et al., Experientia, 40(1984), 738-739.

[14] Hazum, E. and Chang, K. J., Science, 205(1979), 1033-1035.

[15] Sharp, B. M., Endocrinology, 117(1985), 793-795. 